\title{
Ethnomedicinal uses of Sthalavrikshas (temple trees)
in Tamil Nadu, southern India
}

\author{
M. Gunasekaran \& P. Balasubramanian
}

\section{Research}

\begin{abstract}
Worship of plants is practiced throughout the world and is well established from pre-historic periods in India. In Tamil Nadu state, India this customary practice follows with religious faiths and culture. One such religious worship is known as Sthalavriksha (sthal: locality, vriksha: tree) in temples. Very few field studies have been conducted on Sthalavriksha practice and its role in social, ecological and environmental impacts of local people. In particular, ethnomedicinal uses on Sthalavrikshas, occurring in the temples of Tamil Nadu have been unexplored. A survey was conducted at 1165 ancient temples of the state and revealed the occurrence of 112 plant species during 20022006. At the time of study, several ethnomedicinal uses of 101 Sthalavriksha species were recorded by both direct observations and referred to by devotees, priests and Nattuvaidyas (traditional healers) in the temples.
\end{abstract}

\section{Introduction}

Sthalavriksha is referred to as a plant, which is venerated from time immemorial by devotees as being as holy as the presiding deity of a temple (Gunasekaran \& Balasubramanian 2005). The Sanskrit term Sthalavriksha means tree of the locality (Sthal - place; Vriksha - tree). Sthalavriksha is a natural tree found in the temple site before construction of the temple and most temple myths (sthalapuranas) and temple histories (sthalavaralaru) refer to a prime deity that was first unearthed or found under the tree. Sthalavriksha is a single plant mostly in the form of a tree or in some places occurring as an herb, shrub, grass or climber. These are different than a group of plants found in sacred sites or sacred groves. After the construction of temples, these plants are treated as Sthalavriksha or temple trees (sacred plants). Due to traditional beliefs, both the devotees and temple authorities serve as protectors of the Sthalavriksha in temples and if a tree (Sthalavriksha) dies because of old age, it is usually replaced by a sapling from the same species. Hence, the tree occurs constantly in a temple for several centuries. However, in most cases the original Sthalavriksha are still living within the temple grounds. In Hinduism, especially in Shaivam, there are three important aspects of the temple grounds, Moorthy (a Deity), Sthalam, (a Shrine and Sthalavriksha) and a Theertham, (Sacred tank or water body). These are the three prime elements to learn about the antiquity of a temple. The worship of these three elements will yield wisdom even without a guru or teacher (Thambiran 1963). In Tamil Nadu there are 25,000 ancient temples and these heritage sites play a vital role in conserving traditional arts, temple architectures, Tamil culture and also Sthalavrikshas. Sthalavrikshas are an integral part of temple worship and this practice is still in vogue in Tamil Nadu and its bordering states of Kerala, Karnataka, Andhra Pradesh and the neighboring Island nation of Sri Lanka. Ancient history of Tamil Nadu reveals the origin and development of temple tradition in the state through

\section{Correspondence}

M. Gunasekaran, Division of Landscape Ecology, Salim Ali Centre for Ornithology and Natural History, Anaikatty, Coimbatore 641-108, Tamil Nadu, INDIA.

gunasekaranmc@yahoo.co.in.

P. Balasubramanian, Division of Landscape Ecology, Salim Ali Centre for Ornithology and Natural History, Anaikatty, Coimbatore 641-108, Tamil Nadu, INDIA.

Ethnobotany Research \& Applications 10:253-268 (2012)

Published: July 3, 2012 


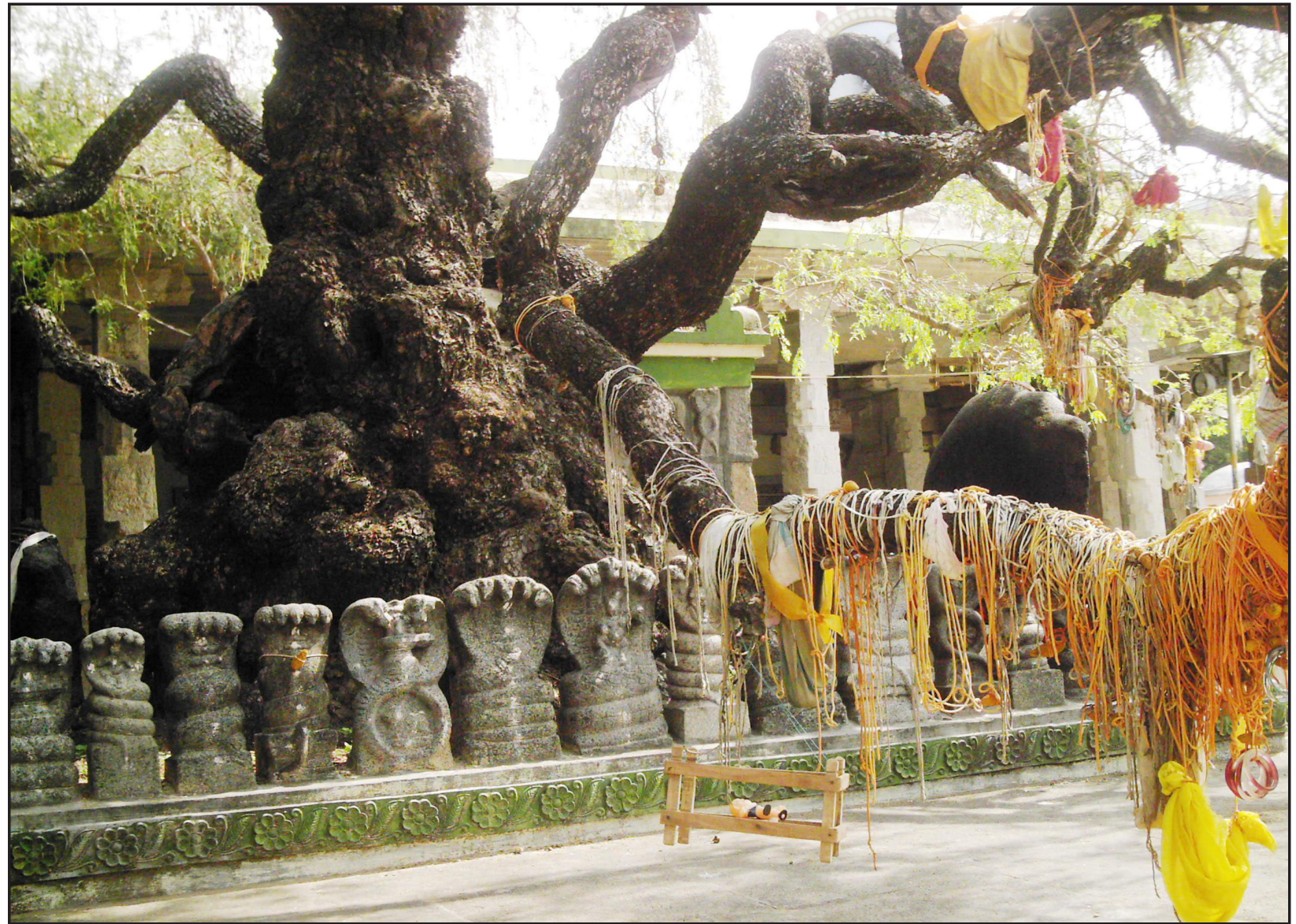

Figure 1. Huge Sthalavrikaha $(\mathrm{gbh}+800 \mathrm{~cm}$ ) Prosopis cineraria (L.) Druce with offerings at Vedaranyam Siva temple, in Tamil Nadu, India.

the epigraphs and ancient Tamil literature, sacred hymns and poems.

Sthalavriksha worship is mostly associated with Shaivam (worship of Lord Siva), Vaishnavam (worship of Lord Vishnu) and Sthalavriksha get divine power from these deities, which are treated as equal to the prime deity of the temple (Nedunchezhiyan 2005, Srinivasan 1972). Many medieval Tamil sacred hymns e.g., Devaram (Thambiran 1997) and Thiviyaprabantham (Anon 1962) refer to Sthalavrikshas and their associated deities. Even though Sthalavriksha worship is an ancient practice in Tamil Nadu very few studies have been conducted on its importance (Amirthalingam 1998, Sundara Sobitharaj 1994, Thirugnanam 1995). In particular, medicinal uses of Sthalavrikshas were referred to, based on secondary sources only.

Hence, the present study was designed to gather data using a field survey:

1. To catalogue all Sthalavriksha species and their associated deities in the temples of Tamil Nadu.
2. To document the ethnomedicinal utilization of user groups including devotees, priests and Nattuvaidiyas on Sthalavrikshas.

\section{Study Areas}

The study was carried out in Tamil Nadu and Puducherry states. Tamil Nadu state is situated at the southernmost corner of the Indian peninsula. It is located between $8^{\circ} 05^{\prime}$ and $13^{\circ} 35^{\prime}$ North latitudes and $76^{\circ} 15^{\prime}$ and $80^{\circ} 20^{\prime}$ East longitudes and covers an area of 1,30,058 $\mathrm{km}^{2}$, occupying $4 \%$ of the total land area of India. Tamil Nadu state has three phytogeographic regions, i.e., East coast, Central plains and Western Ghats. The study was designed to cover all three regions of the state.

\section{Methods}

The survey was conducted in temples that were a minimum of 100 years of age, very few temples were surveyed that were less than 100 years old to find whether the Sthalavriksha practice still continues. Temples sur- 


\section{Gunasekaran \& Balasubramanian - Ethnomedicinal uses of Sthalavrikshas (temple trees) in Tamil Nadu, southern India}

veyed were sorted into age categories: >1000 years, 5001000 years, $100-500$ years, and less than 100 years.

Sthalavriksha specimens were collected in temples at the rate of one specimen from each species for herbarium preparation and identification. Plants were identified on the basis of Flora of the Presidency of Madras (Gamble, reprint 1986), Flora of Tamil Nadu (Henry et al. 1987, 1989, Nair \& Henry 1983). The herbaria at the Botanical Survey of India, Southern Circle and Institute of Forest Genetics and Tree Breeding, Coimbatore were consulted for correct botanical identification. After confirming the identity, the herbarium specimens were deposited in the Herbarium of Salim Ali Centre for Ornithology and Natural History, Coimbatore.

Sthalavrikshas are the common property of the particular temple and the ethnomedicinal knowledge is common to the local society and people living nearby that particular temple belonging to several castes and many ethnic communities (Hindus). No specific permission from authorities was required to learn ethnomedicinal uses from the people in the local communities. During the temple survey the researchers interviewed the local priest about the user groups and from them were selected people over 40 years of age to approach.
Documentation of the medicinal utilization of Sthalavriksha species by user groups were recorded using two techniques:

1. Interview and questionnaire - A questionnaire was used to record the knowledge of the ethnomedicinal use from the user groups, including parts used, disease treated, etc.

2. Direct observation and interaction - Collections of leaves and withered plant parts of Sthalavrikshas by user groups were observed in a few occasions and interactions with these people were done learning about medicinal utilization of the Sthalavrikshas.

\section{Results}

Information regarding the binomial and the local names for each Sthalavrikshas are listed in Table 1. All the information obtained related to medicines prepared for treating different ailments. Interview and questionnaire approaches are provided separately in Table 2. Data related to the second approach (direct observation) including Sthalavriksha species, parts used and diseases treated are furnished in Table 3.

In Tamil Nadu state, eastern and southern regions have a higher number of temples than any other region. A total of 1165 temples, distributed in 30 districts of Tamil Nadu

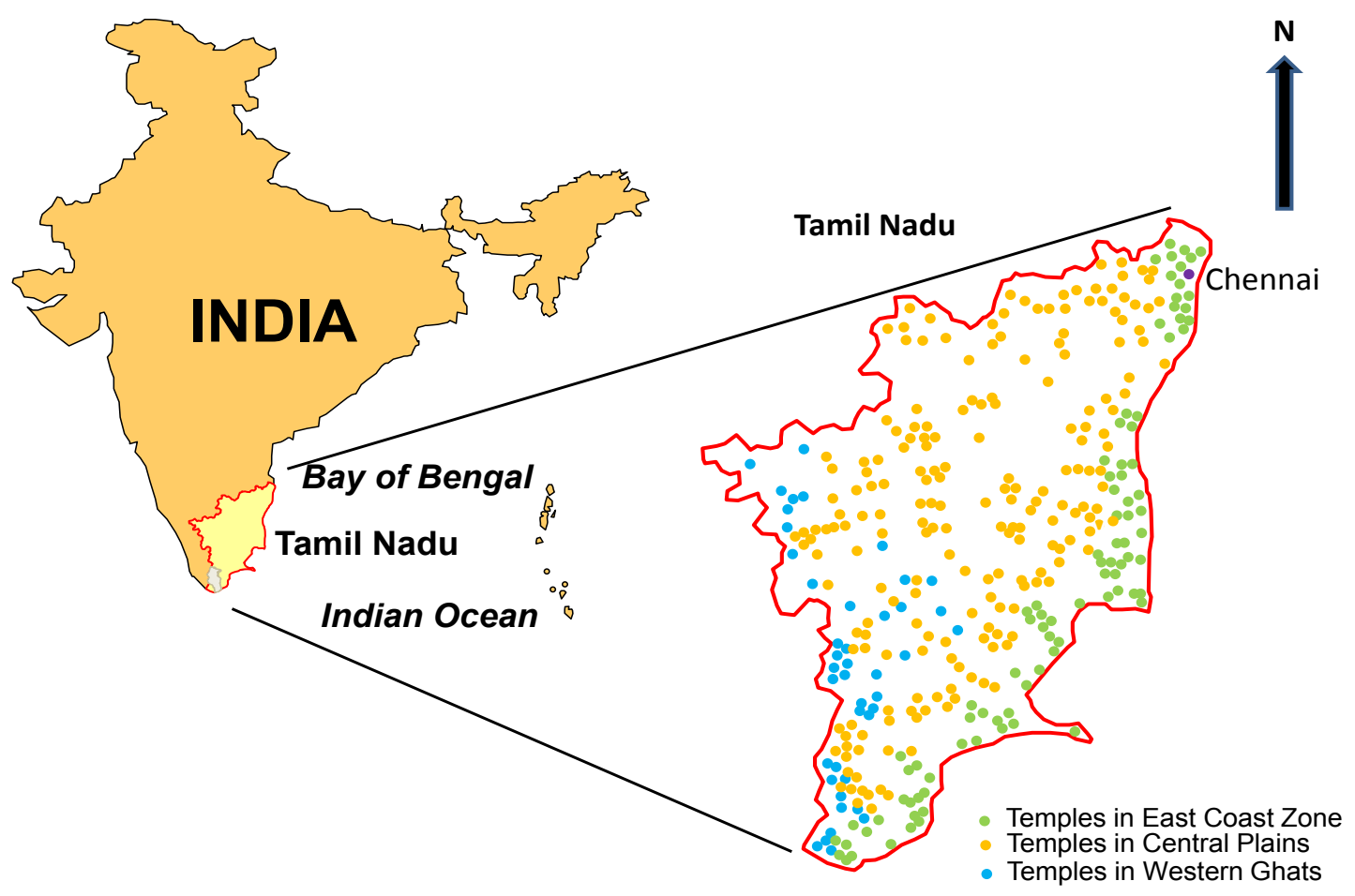

Figure 2. Temples surveyed in three different biogeoraphic zones in Tamil Nadu, India. 
and Puducherry, were surveyed. During the study, temples constructed in the kingdoms of Chera, Chola, Pandia, Pallava, Nayakas and the latest period (20th century) were surveyed. In all the districts except Chennai, a minimum of 30 temples were surveyed. Chennai is a metropolitan city and the smallest district in the state. This district only has a small number of historic temples. Hence, Chennai district and the adjoining Thiruvallur and Kanchipuram districts, only 20 temples were surveyed. The highest number of temples were surveyed in Nagapattinam and Tirunelveli districts viz., 80 and 76 respectively.

A total of 127 people were interviewed after obtaining their oral consent, among them only 11 were women. Most of these ladies were collecting the withered parts of the Sthalavrikshas to ward off evil spirits from their home. Of the remaining 116 people 85 were local devotees, 17 temple priests and 14 were local traditional healers.
A total of 1165 temples surveyed which include 846 Siva temples, 246 Vishnu temples, 23 Amman temples, 48 Murugan temples and two other deity temples. Of 1165 temples surveyed, Sthalavrikshas were present in 820 temples. In total 112 species of Sthalavrikshas belonging to 41 families were recorded (Table 1) during our field research between 2002-2006. Of the 112 Sthalavriksha species documented in temples 83 are trees followed by 17 shrubs, 7 climbers, 3 grasses and 2 herbs. Among the 112 species, Bengal quince (Aegle marmelos (L.) Corrêa) was the most frequently recorded $(n=324)$ at temples followed by Prosopis cineraria (L.) Druce $(n=63)$. The leaves of the $A$. marmelos and $P$. cineraria are used as offerings to Lord Siva and these trees are thought to occur in higher numbers in temples than in the wild in Tamil Nadu. All 112 Sthalavriksha species recorded were angiosperms and 102 species were dicotyledons belonging to 78 genera and 38 families, and ten species of monocotyledons, representing 3 families.

Table 1. List of Sthalavrikshas recorded during a survey of temple trees in Tamil Nadu, India.

\begin{tabular}{|c|c|c|c|}
\hline Scientific Name & Vernacular Name & Habit & $\begin{array}{l}\text { Voucher } \\
\text { No. }\end{array}$ \\
\hline Acacia chundra Willd. (Fabaceae) & Karungali & Tree & 238 \\
\hline Acacia farnesiana (L.) Willd. (Fabaceae) & Odaimaram & Tree & 277 \\
\hline Acacia leucophloea Willd. (Fabaceae) & Velavel & Tree & 10 \\
\hline Aegle marmelos (L.) Corrêa (Rutaceae) & Vilvam & Tree & 3 \\
\hline Alangium salviifolium (L.f.) Wangerin (Cornaceae) & Alangiam & Tree & 293 \\
\hline Albizia amara (Roxb.) Boivin (Fabaceae) & Osilai & Tree & 271 \\
\hline Albizia lebbeck (L.) Benth. (Fabaceae) & Vahai & Tree & 139 \\
\hline Andropogon pumilus Roxb. (Poaceae) & Vizhal & Grass & 263 \\
\hline Artabotrys hexapetalus (L.f.) Bhandari (Annonaceae) & Manoranjitham & Climber & 321 \\
\hline Artocarpus heterophyllus Lam. (Moraceae) & Pala & Tree & 236 \\
\hline Artocarpus hirsutus Lam. (Moraceae) & Ayini & Tree & 289 \\
\hline Atalantia monophylla DC. (Rutaceae) & Kurunthai & Tree & 62 \\
\hline Azadirachta indica A. Juss. (Meliaceae) & Vembu & Tree & 25 \\
\hline Bambusa arundinacea (Retz.) Willd. (Poaceae) & Moongil & Grass & 188 \\
\hline Bauhinia acuminata L. (Fabaceae) & Mantharai & Tree & 242 \\
\hline Bauhinia purpurea L. (Fabaceae) & Mantharai & Tree & 74 \\
\hline Bauhinia racemosa Lam. (Fabaceae) & Mantharai & Tree & 327 \\
\hline Borassus flabellifer L. (Arecaceae) & Panai & Tree & 296 \\
\hline Butea monosperma (Lam.) Taub. (Fabaceae) & Purasu & Tree & 15 \\
\hline Cadaba fruticosa (L.) Druce (Capparaceae) & Vizhi & Shrub & 85 \\
\hline Calamus rotang L. (Arecaceae) & Pirambu & Climber & 46 \\
\hline Calophyllum inophyllum L. (Calophyllaceae) & Punnai & Tree & 124 \\
\hline Calotropis procera (Aiton) W.T. Aiton (Apocynaceae) & Velerukku & Shrub & 292 \\
\hline Canthium parviflorum Lam. (Rubiaceae) & Karai & Tree & 117 \\
\hline Capparis divaricata Lam. (Capparaceae) & Thoratti & Tree & 63 \\
\hline
\end{tabular}



(temple trees) in Tamil Nadu, southern India

\begin{tabular}{|c|c|c|c|}
\hline Scientific Name & Vernacular Name & Habit & \begin{tabular}{|l} 
Voucher \\
No. \\
\end{tabular} \\
\hline Capparis zeylanica L. (Capparaceae) & Peyarillamaram & Tree & 283 \\
\hline Carissa carandas L. (Apocynaceae) & Kala & Shrub & 421 \\
\hline Carissa spinarum L. (Apocynaceae) & Kala & Shrub & 179 \\
\hline Cassia fistula L. (Fabaceae) & Sarakondrai & Tree & 142 \\
\hline Citrus aurantiifolia (Christm.) Swingle (Rutaceae) & Yelumichai & Shrub & 35 \\
\hline Citrus pennivesiculata (Tan.) (Rutaceae) & Narathai & Tree & 41 \\
\hline Cocos nucifera L. (Arecaceae) & Thennai & Tree & 157 \\
\hline Commiphora caudata Engl. (Burseraceae) & Kiluvai & Tree & 47 \\
\hline Cordia domestica Roth (Boraginaceae) & Uthalam & Tree & 169 \\
\hline Corypha umbraculifera L. (Arecaceae) & Thalapanai & Tree & 259 \\
\hline Crateva magna (Lour.) DC. (Capparaceae) & Mavilangam & Tree & 67 \\
\hline Crescentia cujete L. (Bignoniaceae) & Thiruvottukai & Tree & 434 \\
\hline Dichrostachys cinerea (L.) Wight \& Arn. (Fabaceae) & Vedathalan & Shrub & 143 \\
\hline Diospyros montana Roxb. (Ebenaceae) & Vakkanai & Tree & 56 \\
\hline Dodonaea viscosa Jacq. (Sapindaceae) & Virali & Shrub & 7 \\
\hline Ehretia ovalifolia Wight (Boraginaceae) & Karukattan & Tree & 164 \\
\hline Ensete edule Bruce ex Horan. (Musaceae) & Monthan & Tree & 187 \\
\hline Euphorbia nivulia Buch.-Ham. (Euphorbiaceae) & Ilaikalli & Tree & 83 \\
\hline Excoecaria agallocha L. (Euphorbiaceae) & Thillai & Tree & 407 \\
\hline Ficus racemosa L. (Moraceae) & Athi & Tree & 397 \\
\hline Ficus benghalensis L. (Moraceae) & Aal & Tree & 171 \\
\hline Ficus microcarpa L.f. (Moraceae) & Kallal & Tree & 307 \\
\hline Ficus mollis Vahl (Moraceae) & Kalathi & Tree & 330 \\
\hline Ficus nervosa B. Heyne ex Roth (Moraceae) & Selamaram & Tree & 417 \\
\hline Ficus religiosa L. (Moraceae) & Arasu & Tree & 468 \\
\hline Ficus virens Dryand. (Moraceae) & Ithi & Tree & 115 \\
\hline Guettarda speciosa L. (Rubiaceae) & Panneer & Tree & 339 \\
\hline Holoptelea integrifolia Planch. (Ulmaceae) & Aacha & Tree & 376 \\
\hline Imperata cylindrica var. major (Nees) C.E. Hubb. (Poaceae) & Tharupai & Grass & 175 \\
\hline Jasminum auriculatum Vahl (Oleaceae) & Mullai & Climber & 87 \\
\hline Jasminum cuspidatum Rottl. \& Willd. (Oleaceae) & Mullai & Climber & 141 \\
\hline Jasminum grandiflorum L. (Oleaceae) & Jathimalli & Climber & 312 \\
\hline Jasminum sambac (L.) Aiton (Oleaceae) & Malligai & Climber & 239 \\
\hline Lepisanthes tetraphylla (Vahl) Radlk. (Sapindaceae) & Neikotta & Tree & 172 \\
\hline Limonia acidissima L. (Rutaceae) & Vila & Tree & 280 \\
\hline Madhuca longifolia (J. König ex L.) J.F. Macbr. (Sapotaceae) & Ilupai & Tree & 61 \\
\hline Magnolia grandiflora L. (Magnoliaceae) & Malaimagudam & Tree & 95 \\
\hline Mangifera indica L. (Anacardiaceae) & Ma & Tree & 55 \\
\hline Manilkara hexandra (Roxb.) Dubard (Sapotaceae) & Paala & Tree & 77 \\
\hline Michelia champaca L. (Magnoliaceae) & Senpagam & Tree & 125 \\
\hline Millingtonia hortensis L. f. (Bignoniaceae) & Maramalli & Tree & 284 \\
\hline
\end{tabular}




\begin{tabular}{|c|c|c|c|}
\hline Scientific Name & Vernacular Name & Habit & $\begin{array}{l}\text { Voucher } \\
\text { No. }\end{array}$ \\
\hline Mimosa pudica L.( Fabaceae) & Thottachinungi & Herb & 329 \\
\hline Mimusops elengi L. (Sapotaceae) & Mahizham & Tree & 439 \\
\hline Morinda pubescens Sm. (Rubiaceae) & Manjanathi & Tree & 415 \\
\hline Moringa oleifera Lam. (Moringaceae) & Murungai & Tree & 36 \\
\hline Murraya koenigii (L.) Spreng. (Rutaceae) & Karuveppilai & Shrub & 370 \\
\hline Musa paradisiaca L. (Musaceae) & Vazhai & Tree & 279 \\
\hline Naringi crenulata Nicolson (Rutaceae) & Mahavilvam & Tree & 51 \\
\hline Neolamarckia cadamba (Roxb.) Bosser (Rubiaceae) & Kadampu & Tree & 428 \\
\hline Nerium oleander L. (Apocynaceae) & Arali & Shrub & 385 \\
\hline Nyctanthes arbor-tristis L. (Oleaceae) & Pavazhamalli & Tree & 97 \\
\hline $\begin{array}{l}\text { Ochna obtusata DC. var. gamblei (King ex Brandis) Kanis (Ochna- } \\
\text { ceae) }\end{array}$ & Silanthi & Tree & 387 \\
\hline Ocimum tenuiflorum L. (Lamiaceae) & Thulasi & Herb & 210 \\
\hline Phoenix sylvestris (L.) Roxb. (Arecaeae) & Icham & Tree & 112 \\
\hline Phyllanthus emblica L. (Phyllanthaceae) & Nelli & Tree & 131 \\
\hline Pleiospermium alatum (Wight \& Arn.) Swingle (Rutaceae) & Kurunthai & Tree & 462 \\
\hline Pongamia pinnata (L.) Pierre (Fabaceae) & Pungam & Tree & 17 \\
\hline Premna latifolia Roxb. (Lamiaceae) & Kattuminnai & Tree & 39 \\
\hline Prosopis cineraria (L.) Druce (Fabaceae) & Vanni & Tree & 267 \\
\hline Pterocarpus marsupium Roxb. (Fabaceae) & Vengai & Tree & 422 \\
\hline Punica granatum L. (Lythraceae) & Madulai & Shrub & 89 \\
\hline Ricinus communis L. (Euphorbiaceae) & Amanaku & Shrub & 159 \\
\hline Salvadora persica L. (Salvadoraceae) & Kalar Ugai & Tree & 226 \\
\hline Santalum album L. (Santalaceae) & Santhanam & Tree & 294 \\
\hline Saraca asoca (Roxb.) De Wilde. (Fabaceae) & Asokam & Tree & 463 \\
\hline Schleichera oleosa (Lour.) Oken (Sapindaceae) & Poovan & Tree & 65 \\
\hline Scaevola plumieri (L). Vahl (Goodeniaceae) & Rutharacham & Shrub & 189 \\
\hline Securinega leucopyrus (Willd.) Müll. Arg. (Phyllanthaceae) & Venpoola & Shrub & 340 \\
\hline Stereospermum chelonoides DC. (Bignoniaceae) & Pathiri & Tree & 172 \\
\hline $\begin{array}{l}\text { Stereospermum colais (Buch.-Ham. ex Dillwyn) Mabb. (Bignoniace- } \\
\text { ae) }\end{array}$ & Pathiri & Tree & 357 \\
\hline Stobilanthes kunthiana (Nees) T. And. (Acanthaceae) & Kurunji & Shrub & 82 \\
\hline Streblus asper Lour. (Moraceae) & Parai & Tree & 209 \\
\hline Strychnos nux-vomica L. (Loganiaceae) & Yetti & Tree & 328 \\
\hline Strychnos potatorum L.f. (Loganiaceae) & Thettra & Tree & 432 \\
\hline Syzygium cumini (L.) Skeels (Myrtaceae) & Naval & Tree & 29 \\
\hline $\begin{array}{l}\text { Tabernaemontana divaricata (L.) R. Br. ex Roem. \& Schulte. (Apocy- } \\
\text { naceae) }\end{array}$ & Nanthiavattai & Shrub & 137 \\
\hline Tabernaemontana heyneana Wall. (Apocynaceae) & Nanthiavattai & Shrub & 447 \\
\hline Tamarindus indica L. (Fabaceae) & Puli & Tree & 218 \\
\hline Tarenna asiatica Kuntz ex K. Schum. (Rubiaceae) & Kura & Tree & 423 \\
\hline Telosma minor (Andrews) W.G. Craib (Apocynaceae) & Sambangi & Climber & 459 \\
\hline
\end{tabular}




\section{Gunasekaran \& Balasubramanian - Ethnomedicinal uses of Sthalavrikshas (temple trees) in Tamil Nadu, southern India}

\begin{tabular}{|l|l|l|l|}
\hline Scientific Name & Vernacular Name & Habit & $\begin{array}{l}\text { Voucher } \\
\text { No. }\end{array}$ \\
\hline Terminalia arjuna (Roxb. ex DC.) Wight \& Arn. (Combretaceae) & Marutham & Tree & 336 \\
\hline Terminalia bellirica (Gaertn.) Roxb. (Combretaceae) & Thani & Tree & 224 \\
\hline Terminalia catappa L. (Combretaceae) & Badam & Tree & 461 \\
\hline Terminalia chebula Retz. (Combretaceae) & Kadukkai & Tree & 190 \\
\hline Vitex negundo L. (Lamiaceae) & Nochi & Shrub & 158 \\
\hline Wrightia tinctoria R. Br. (Apocynaceae) & Palai & Tree & 358 \\
\hline Ziziphus mauritiana Lam. (Rhamnaceae) & llandai & Tree & 31 \\
\hline
\end{tabular}

\section{Medicinal uses}

The devotees and local traditional medical practitioners (Nattu Vaidyas) use several Sthalavriksha plants for treating various ailments. Normally, the priests or the Vaidyas prescribe medicines with devotion and devotees consume the medicines with great belief. Most of the plants are said to contain medicinal properties (Anon 1988-89). A. marmelos is the most utilized Sthalavriksha followed by Azadirachta indica A. Juss., Ocimum tenuiflorum L. and Naringi crenulata Nicolson. The villagers know the medicinal properties of the plant resources; hence medicinal utilization of Sthalavriksha species is probably more common in the villages than in the cities. Two factors pertain to these practices: 1) traditional beliefs and 2) devotional approaches to the Sthalavrikshas in tem- ples. Generally villagers treat the Sthalavrikshas as living gods of the temples, whereas the prime deity is in the form of stone statues. Additionally, Sthalavriksha medicines are cheaper or are free (as an offering to a priest) and ease of access to these is a factor that maintains this practice by the local communities.

In addition to 91 Sthalavriksha medicinal uses discussed by users (Table 2), the collection of leaves and withered plant parts of 11 species were also documented from temples (Table 3).

The Nattu-Vaidiyas and local devotees collected these plant parts for medicinal purposes. Women often collect these materials based on local beliefs, for instance to tie the plant parts at their doorsteps to ward off evil spirits.

Table 2. Medicinal uses of Sthalavrikshas reported by temple priests, devotees, and local Nattu Vaidiya in response to a questionnaire, in Tamil Nadu, India.

\begin{tabular}{|c|c|c|c|}
\hline $\begin{array}{l}\text { Sthala- } \\
\text { vrikshas } \\
\text { No. }\end{array}$ & Sthalavriksha & Parts Used & Disease Treated \\
\hline 1. & Acacia chundra Willd. (Fabaceae) & Bark & Tooth ache \\
\hline \multirow[t]{2}{*}{2.} & \multirow[t]{2}{*}{ Acacia leucophloea Willd. (Fabaceae) } & Bark & Tooth ache \\
\hline & & Gum & Tooth ache \\
\hline \multirow[t]{2}{*}{3.} & \multirow[t]{2}{*}{ Alangium salviifolium (L.f.) Wangerin (Cornaceae) } & Bark & Antidote \\
\hline & & Seeds & Laxative \\
\hline 4. & Albizia amara (Roxb.) Boivin (Fabaceae) & Leaves & Dandruff \\
\hline \multirow[t]{3}{*}{5.} & \multirow[t]{3}{*}{ Albizia lebbeck (L.) Benth. (Fabaceae) } & Bark & Tooth ache \\
\hline & & Leaves & Antidote \\
\hline & & Seeds & Eye diseases \\
\hline 6. & Andropogon pumilus Roxb. (Poaceae) & Roots & Joint pain \\
\hline \multirow[t]{2}{*}{7.} & \multirow[t]{2}{*}{ Artocarpus heterophyllus Lam. (Moraceae) } & Leaves & Skin diseases, Ulcer \\
\hline & & Root & Asthma, Diarrhea \\
\hline 8. & Artocarpus hirsutus Lam. (Moraceae) & Leaves & Bubonic plague \\
\hline \multirow[t]{3}{*}{9.} & \multirow[t]{3}{*}{ Atalantia monophylla DC. (Rutaceae) } & Leaves & Itching \\
\hline & & Root & Joint pain \\
\hline & & Oil & Paralysis \\
\hline
\end{tabular}




\begin{tabular}{|c|c|c|c|}
\hline $\begin{array}{l}\text { Sthala- } \\
\text { vrikshas } \\
\text { No. }\end{array}$ & Sthalavriksha & Parts Used & Disease Treated \\
\hline \multirow[t]{7}{*}{10.} & \multirow[t]{7}{*}{ Azadirachta indica A. Juss. (Meliaceae) } & Bark & Astringent, Leprosy \\
\hline & & Leaves & Antiseptic, Measles \\
\hline & & Flowers & Liver diseases \\
\hline & & Fruit & Cut wounds \\
\hline & & Root & Antidote \\
\hline & & Seed & Dandruff \\
\hline & & Oil & Laxative, Ringworm \\
\hline 11. & Bambusa arundinacea (Retz.) Willd. (Poaceae) & Leaves & $\begin{array}{l}\text { Cooling tonic, Asthma, } \\
\text { Cough }\end{array}$ \\
\hline \multirow[t]{4}{*}{12.} & \multirow[t]{4}{*}{ Bauhinia acuminata L. (Fabaceae) } & Bark & Bladder stone \\
\hline & & Leaves & Leprosy, Asthma \\
\hline & & Flowers & Cooling effect \\
\hline & & Root & Burns \\
\hline 13. & Bauhinia purpurea L. (Fabaceae) & Bark & Ulcer \\
\hline \multirow[t]{2}{*}{14.} & \multirow[t]{2}{*}{ Borassus flabellifer L. (Arecaceae) } & Flower Juice & Refrigerant \\
\hline & & Fruit & Boils, Diarrhoea \\
\hline 15. & Butea monosperma (Lam.) Taub. (Fabaceae) & Seeds & Ringworm \\
\hline 16. & Cadaba fruticosa (L.) Druce (Capparaceae) & Leaves & Intestinal worms \\
\hline 17. & Calamus rotang L. (Arecaceae) & Tuber & Cold, Cough, Fever \\
\hline \multirow[t]{3}{*}{18.} & \multirow[t]{3}{*}{ Calophyllum inophyllum L. (Calophyllaceae) } & Bark & Bleeding \\
\hline & & Leaves & Eye diseases \\
\hline & & Flowers & Fever \\
\hline \multirow[t]{2}{*}{19.} & \multirow[t]{2}{*}{ Calotropis procera (Aiton) W.T. Aiton (Apocynaceae) } & Bark & Dysentery \\
\hline & & Flowers & Cold, Cough, Asthma \\
\hline \multirow[t]{2}{*}{20.} & \multirow[t]{2}{*}{ Canthium parviflorum Lam. (Rubiaceae) } & Leaves & Dysentery, Diarrhea \\
\hline & & Flowers & Refrigerant \\
\hline \multirow[t]{2}{*}{21.} & \multirow[t]{2}{*}{ Capparis zeylanica L. (Capparaceae) } & Bark & Fever \\
\hline & & Leaves & Stomach problems \\
\hline \multirow[t]{2}{*}{22.} & \multirow[t]{2}{*}{ Carissa carandas L. (Apocynaceae) } & Flowers & Eye diseases \\
\hline & & Fruit & Vomiting, Dropsy \\
\hline \multirow[t]{2}{*}{23.} & \multirow[t]{2}{*}{ Carissa spinarum L. (Apocynaceae) } & Flowers & Eye diseases \\
\hline & & Fruit & Vomiting, Dropsy \\
\hline \multirow[t]{2}{*}{24.} & \multirow[t]{2}{*}{ Cassia fistula L. (Fabaceae) } & Bark & Laxative, Fever \\
\hline & & Fruit & Laxative \\
\hline \multirow[t]{2}{*}{25.} & \multirow[t]{2}{*}{ Citrus aurantiifolia (Christm.) Swingle (Rutaceae) } & Fruit & Scurvy, Dysentery \\
\hline & & Oil & Stimulant \\
\hline \multirow[t]{2}{*}{26.} & \multirow[t]{2}{*}{ Citrus pennivesiculata Tan. (Rutaceae) } & Fruit & Dysentery \\
\hline & & Oil & Stimulant \\
\hline \multirow[t]{2}{*}{27.} & \multirow[t]{2}{*}{ Cocos nucifera L. (Arecaceae) } & Tender Fruit & Coolant \\
\hline & & Flower juice & $\begin{array}{l}\text { Stimulant, Natural Vin- } \\
\text { egar }\end{array}$ \\
\hline
\end{tabular}




\section{Gunasekaran \& Balasubramanian - Ethnomedicinal uses of Sthalavrikshas (temple trees) in Tamil Nadu, southern India}

\begin{tabular}{|c|c|c|c|}
\hline $\begin{array}{l}\text { Sthala- } \\
\text { vrikshas } \\
\text { No. }\end{array}$ & Sthalavriksha & Parts Used & Disease Treated \\
\hline \multirow[t]{2}{*}{28.} & \multirow[t]{2}{*}{ Commiphora caudata Engl. (Burseraceae) } & Bark & Diarrhea \\
\hline & & Leaves & Dysentery \\
\hline 29. & Cordia domestica Roth (Boraginaceae) & Bark & $\begin{array}{l}\text { Stomach ache, Mouth } \\
\text { Ulcer }\end{array}$ \\
\hline \multirow[t]{2}{*}{30.} & \multirow[t]{2}{*}{ Crateva magna (Lour.) DC. (Capparaceae) } & Bark & Cleaning \\
\hline & & Leaves & Fever \\
\hline \multirow[t]{2}{*}{31.} & \multirow[t]{2}{*}{ Crescentia cujete L. (Bignoniaceae) } & Bark & Laxative, Ulcer \\
\hline & & Fruit & Refrigerant \\
\hline 32. & Dichrostachys cinerea (L.) Wight \& Arn. (Fabaceae) & Leaves & Eye complaints \\
\hline 33. & Diospyros montana Roxb. (Ebenaceae) & Fruit & Boils \\
\hline 34. & Dodonaea viscosa Jacq. (Sapindaceae) & Leaves & $\begin{array}{l}\text { Wounds, Swelling, } \\
\text { Burns }\end{array}$ \\
\hline \multirow[t]{4}{*}{35.} & \multirow[t]{4}{*}{ Ensete edule Bruce ex Horan. (Musaceae) } & Stem Juice & $\begin{array}{l}\text { Peptic Ulcer, Bladder } \\
\text { stone }\end{array}$ \\
\hline & & Bark & Burn ulcer \\
\hline & & Flowers & Dysentery, Diarrhoea \\
\hline & & Fruit & Ulcer \\
\hline \multirow[t]{3}{*}{36.} & \multirow[t]{3}{*}{ Euphorbia nivulia Buch.-Ham. (Euphorbiaceae) } & Bark & Boils \\
\hline & & Stem & Ulcers in nails \\
\hline & & Latex & $\begin{array}{l}\text { Knee and joint pain, } \\
\text { Laxative }\end{array}$ \\
\hline \multirow[t]{3}{*}{37.} & \multirow[t]{3}{*}{ Excoecaria agallocha L. (Euphorbiaceae) } & Flowers & Eye diseases \\
\hline & & Root & $\begin{array}{l}\text { Tooth ache, Intestinal } \\
\text { worms }\end{array}$ \\
\hline & & Oil & Joint pain, Leprosy \\
\hline \multirow[t]{4}{*}{38.} & \multirow[t]{4}{*}{ Ficus benghalensis L. (Moraceae) } & Bark & Dysentery \\
\hline & & Leaves & Diarrhea \\
\hline & & Seeds & Refrigerant \\
\hline & & Latex & Pains, Bruises \\
\hline 39. & Ficus microcarpa L. f. (Moraceae) & Bark & Fever \\
\hline 40. & Ficus mollis Vahl (Moraceae) & Bark & Urinary infections \\
\hline \multirow[t]{5}{*}{41.} & \multirow[t]{5}{*}{ Ficus racemosa L. (Moraceae) } & Bark & Ulcer \\
\hline & & Fruit & Laxative \\
\hline & & Leaves & Bilious infections \\
\hline & & Root & Dysentery \\
\hline & & Seeds & Refrigerant \\
\hline \multirow[t]{5}{*}{42.} & \multirow[t]{5}{*}{ Ficus religiosa L. (Moraceae) } & Bark & Ulcer \\
\hline & & Fruit & Laxative \\
\hline & & Latex & Piles, Diarrhea \\
\hline & & Leaves & Cut wounds \\
\hline & & Seeds & Refrigerant \\
\hline 43. & Ficus virens Dryand. (Moraceae) & Seeds & Refrigerant \\
\hline
\end{tabular}




\begin{tabular}{|c|c|c|c|}
\hline $\begin{array}{l}\text { Sthala- } \\
\text { vrikshas } \\
\text { No. }\end{array}$ & Sthalavriksha & Parts Used & Disease Treated \\
\hline 44. & Guettarda speciosa L. (Rubiaceae) & Bark & Dysentery, Cut wounds \\
\hline 45. & Holoptelea integrifolia Planch. (Ulmaceae) & Bark & Rheumatism \\
\hline \multirow[t]{2}{*}{46.} & \multirow{2}{*}{$\begin{array}{l}\text { Imperata cylindrica var. major (Nees) C.E. Hubb. (Poa- } \\
\text { ceae) }\end{array}$} & Leaves & Sedative \\
\hline & & Root & Diarrhoea \\
\hline \multirow[t]{2}{*}{47.} & \multirow[t]{2}{*}{ Jasminum auriculatum Vahl (Oleaceae) } & Leaves & $\begin{array}{l}\text { Ulcers in mouth and } \\
\text { throat }\end{array}$ \\
\hline & & Flowers & $\begin{array}{l}\text { Eye diseases, Head- } \\
\text { ache }\end{array}$ \\
\hline \multirow[t]{2}{*}{48.} & \multirow[t]{2}{*}{ Jasminum cuspidatum Rottl. (Oleaceae) } & Flowers & Ulcer \\
\hline & & Leaves & Fever \\
\hline 49. & Jasminum grandiflorum L. (Oleaceae) & Flowers & Ulcer \\
\hline \multirow[t]{2}{*}{50.} & \multirow[t]{2}{*}{ Jasminum sambac (L.) Aiton (Oleaceae) } & Leaves & Fever \\
\hline & & Flowers & Swelling in testes \\
\hline 51. & Lepisanthes tetraphylla (Vahl) Radlk. (Sapindaceae) & Seeds & Dandruff \\
\hline \multirow[t]{3}{*}{52.} & \multirow[t]{3}{*}{ Limonia acidissima L. (Rutaceae) } & Bark & Vomiting \\
\hline & & Leaves & $\begin{array}{l}\text { Cough, Cold, Prickle } \\
\text { heat boils }\end{array}$ \\
\hline & & Fruit & $\begin{array}{l}\text { Ulcers in throat and } \\
\text { mouth }\end{array}$ \\
\hline 53. & $\begin{array}{l}\text { Madhuca longifolia (J. König ex L.) J.F. Macbr. (Sapo- } \\
\text { taceae) }\end{array}$ & Bark & Skin diseases \\
\hline \multirow[t]{4}{*}{54.} & \multirow[t]{4}{*}{ Mangifera indica L. (Anacardiaceae) } & Bark & $\begin{array}{l}\text { Vomiting, Cracks on } \\
\text { foot }\end{array}$ \\
\hline & & Leaves & $\begin{array}{l}\text { Dysentery, Diarrhea, } \\
\text { Throat pain }\end{array}$ \\
\hline & & Seed & Diarrhea \\
\hline & & Gum & Cracks on foot, Ulcer \\
\hline \multirow[t]{3}{*}{55.} & \multirow[t]{3}{*}{ Michelia champaca L. (Magnoliaceae) } & Bark & Cold and fever, Boils \\
\hline & & Leaves & Stomach ache \\
\hline & & Flowers & $\begin{array}{l}\text { Fever, Urinary prob- } \\
\text { lems }\end{array}$ \\
\hline 56. & Mimosa pudica L.( Fabaceae) & Leaves & Cut wounds \\
\hline \multirow[t]{4}{*}{57.} & \multirow[t]{4}{*}{ Mimusops elengi L. (Sapotaceae) } & Bark & $\begin{array}{l}\text { Tooth ache, Uterus } \\
\text { problems }\end{array}$ \\
\hline & & Leaves & Tooth ache \\
\hline & & Fruit & Tooth ache \\
\hline & & Seeds & Male sterility \\
\hline \multirow[t]{4}{*}{58.} & \multirow[t]{4}{*}{ Morinda pubescens Sm. (Rubiaceae) } & Leaves & Scabies, Ulcer \\
\hline & & Fruit & Tooth ache \\
\hline & & Root & Sedative \\
\hline & & Oil & Skin diseases \\
\hline \multirow[t]{2}{*}{59.} & \multirow[t]{2}{*}{ Moringa oleifera Lam. (Moringaceae) } & Bark & Fever, Fits \\
\hline & & Leaves & Laxative \\
\hline
\end{tabular}




\section{Gunasekaran \& Balasubramanian - Ethnomedicinal uses of Sthalavrikshas (temple trees) in Tamil Nadu, southern India}

\begin{tabular}{|c|c|c|c|}
\hline \begin{tabular}{|l|} 
Sthala- \\
vrikshas \\
No. \\
\end{tabular} & Sthalavriksha & Parts Used & Disease Treated \\
\hline \multirow[t]{3}{*}{ 59. Con't } & \multirow[t]{3}{*}{ Moringa oleifera Lam. (Moringaceae) } & Flowers & Cough, Male sterility \\
\hline & & Fruit & Infertility \\
\hline & & Gum & Male sterility \\
\hline \multirow[t]{2}{*}{60.} & \multirow[t]{2}{*}{ Murraya koenigii (L.) Spreng. (Rutaceae) } & Leaves & Indigestion \\
\hline & & Fruit & $\begin{array}{l}\text { Increasing blood secre- } \\
\text { tion }\end{array}$ \\
\hline \multirow[t]{4}{*}{61.} & \multirow[t]{4}{*}{ Musa acuminata X balbisiaiana Colla } & Stem Juice & $\begin{array}{l}\text { Peptic Ulcer, Bladder } \\
\text { Stone }\end{array}$ \\
\hline & & Bark & Burn, Ulcer \\
\hline & & Flowers & Dysentery, Diarrhea \\
\hline & & Fruit & Ulcer \\
\hline \multirow[t]{2}{*}{62.} & \multirow[t]{2}{*}{ Naringi crenulata Nicolson (Rutaceae) } & Bark & Fever \\
\hline & & Leaves & Cold, Cough \\
\hline 63. & Nerium oleander L. (Apocynaceae) & Bark & Ear pain \\
\hline \multirow[t]{3}{*}{64.} & \multirow[t]{3}{*}{ Nyctanthes arbor-tristis L. (Oleaceae) } & Bark & Skin diseases \\
\hline & & Seeds & Scabies, Herpes \\
\hline & & Leaves & $\begin{array}{l}\text { Fever, Back-pain, In- } \\
\text { testine worms }\end{array}$ \\
\hline \multirow[t]{5}{*}{65.} & \multirow[t]{5}{*}{ Ocimum tenuiflorum L. (Lamiaceae) } & Bark & Fever \\
\hline & & Leaves & $\begin{array}{l}\text { Cold, Fever, Ear pain, } \\
\text { Chest pain }\end{array}$ \\
\hline & & Flowers & Cough \\
\hline & & Seed & Heat diseases \\
\hline & & Root & Fever \\
\hline 66. & Phoenix sylvestris (L.) Roxb. (Arecaeae) & Root & Tooth ache \\
\hline \multirow[t]{3}{*}{67.} & \multirow[t]{3}{*}{ Phyllanthus emblica L. (Phyllanthaceae) } & Leaves & Mouth ulcer \\
\hline & & Fruit & Fever, Blood pressure \\
\hline & & Root bark & Tongue ulcer \\
\hline \multirow[t]{4}{*}{68.} & \multirow[t]{4}{*}{ Pongamia pinnata (L.) Pierre (Fabaceae) } & Oil & Scabies, Leucoderma \\
\hline & & Leaves & Fever \\
\hline & & Flowers & Skin diseases \\
\hline & & Root & Skin diseases \\
\hline 69. & Premna latifolia Roxb. (Lamiaceae) & Leaves & Diuretic \\
\hline \multirow[t]{4}{*}{70.} & \multirow[t]{4}{*}{ Prosopis cineraria (L.) Druce (Fabaceae) } & Bark & Tooth ache \\
\hline & & Leaves & Cold, Cough, Fever \\
\hline & & Flowers & Prevent miscarriage \\
\hline & & Gum & Dysentery \\
\hline \multirow[t]{3}{*}{71.} & \multirow[t]{3}{*}{ Pterocarpus marsupium Roxb. (Fabaceae) } & Bark & Tooth ache \\
\hline & & Flowers & Fever \\
\hline & & Gum & Tooth ache \\
\hline 72. & Punica granatum L. (Lythraceae) & Fruit & Dysentery, Diarrhea \\
\hline
\end{tabular}




\begin{tabular}{|c|c|c|c|}
\hline $\begin{array}{l}\text { Sthala- } \\
\text { vrikshas } \\
\text { No. }\end{array}$ & Sthalavriksha & Parts Used & Disease Treated \\
\hline \multirow[t]{2}{*}{73.} & \multirow[t]{2}{*}{ Ricinus communis L. (Euphorbiaceae) } & Leaves & Stomach ache \\
\hline & & Oil & Sedative \\
\hline 74. & Salvadora persica L. (Salvadoraceae) & Leaves & Diabetics \\
\hline 75. & Santalum album L. (Santalaceae) & Wood & $\begin{array}{l}\text { Pimples, Urinary infec- } \\
\text { tions }\end{array}$ \\
\hline \multirow[t]{2}{*}{76.} & \multirow[t]{2}{*}{ Saraca asoca (Roxb.) De Wilde (Fabaceae) } & Bark & Skin diseases \\
\hline & & Flowers & Dysentery, Diarrhoea \\
\hline 77. & Schleichera oleosa (Lour.) Oken (Sapindaceae) & Fruit & Refrigerant \\
\hline 78. & $\begin{array}{l}\text { Securinega leucopyrus (Willd.) Müll. Arg. (Phyllantha- } \\
\text { ceae) }\end{array}$ & Leaves & Warms sores \\
\hline 79. & Stereospermum chelonoides DC. (Bignoniaceae) & Flowers & Diabetic boils \\
\hline 80. & $\begin{array}{l}\text { Stereospermum colais (Buch.-Ham. ex Dillwyn) Mabb. } \\
\text { (Bignoniaceae) }\end{array}$ & Flowers & Ring worm \\
\hline \multirow[t]{3}{*}{81.} & \multirow[t]{3}{*}{ Streblus asper Lour. (Moraceae) } & Bark & Antidote \\
\hline & & Leaves & Dysentery, Diarrhea \\
\hline & & Latex & $\begin{array}{l}\text { Crack in foot, Tooth } \\
\text { ache }\end{array}$ \\
\hline 82. & Strychnos nux-vomica L. (Loganiaceae) & Leaves & Body boils \\
\hline \multirow[t]{2}{*}{83.} & \multirow[t]{2}{*}{ Strychnos potatorum L.f. (Loganiaceae) } & Bark & Cholera \\
\hline & & Leaves & Ulcers \\
\hline \multirow[t]{3}{*}{84.} & \multirow[t]{3}{*}{ Syzygium cumini (L.) Skeels. (Myrtaceae) } & Leaves & Dysentery, Diarrhea \\
\hline & & Fruit & Reduce blood sugar \\
\hline & & Seed & Diabetes \\
\hline \multirow[t]{2}{*}{85.} & \multirow{2}{*}{$\begin{array}{l}\text { Tabernaemontana divaricata (L.) R. Br. ex Roem. \& } \\
\text { Schulte. (Apocynaceae) }\end{array}$} & Flowers & Eye diseases \\
\hline & & Root & Tooth ache \\
\hline \multirow[t]{5}{*}{86.} & \multirow[t]{5}{*}{ Tamarindus indica L. (Fabaceae) } & Bark & Peptic ulcer \\
\hline & & Leaves & $\begin{array}{l}\text { Stomach ache, Diar- } \\
\text { rhea }\end{array}$ \\
\hline & & Flowers & Eye pain \\
\hline & & Fruit & Uterus problems \\
\hline & & Seed & $\begin{array}{l}\text { Vomiting, Dysentery, } \\
\text { Tooth ache }\end{array}$ \\
\hline 87. & Tarenna asiatica Kuntz ex K. Schum. (Rubiaceae) & Leaves & Antidote \\
\hline 88. & Telosma minor (Andrews) W.G. Craib (Apocynaceae) & Flowers & Refrigerant \\
\hline 89. & Terminalia catappa L. (Combretaceae) & Gum & Cough, Dysentery \\
\hline \multirow[t]{2}{*}{90.} & \multirow[t]{2}{*}{ Terminalia chebula Retz. (Combretaceae) } & Seed & $\begin{array}{l}\text { Eye disease, Stomach } \\
\text { ache }\end{array}$ \\
\hline & & Fruit & Cold, Cough \\
\hline \multirow[t]{3}{*}{91.} & \multirow[t]{3}{*}{ Wrightia tinctoria . Br. (Apocynaceae) } & Bark & $\begin{array}{l}\text { Fever, Dysentery, Diar- } \\
\text { rhea }\end{array}$ \\
\hline & & Leaves & Tooth ache \\
\hline & & Seed & Infertility, Dysentery \\
\hline
\end{tabular}




\section{Gunasekaran \& Balasubramanian - Ethnomedicinal uses of Sthalavrikshas (temple trees) in Tamil Nadu, southern India}

Table 3. Collection of Sthalavrikshas parts observed from temples for medicinal uses.

\begin{tabular}{|c|c|c|c|}
\hline Sthalavriksha & Parts Collected & Parts Used & Disease Treated \\
\hline Acacia farnesiana (L.) Willd. (Fabaceae) & Withered Leaves & Leaf paste & Knee and Joint pain, Infertility \\
\hline \multirow[t]{8}{*}{ Aegle marmelos (L.) Corrêa (Rutaceae) } & Withered Leaves & Leaves & Blood sugar reduction \\
\hline & \multirow[t]{3}{*}{ Fallen Fruits } & Fruit pulp & Skin boils \\
\hline & & Fruit pulp with milk & Diarrhea \\
\hline & & Fruits & $\begin{array}{l}\text { Vilvadhilegium prepared by lo- } \\
\text { cal Vaidyas }\end{array}$ \\
\hline & Withered Leaves & $\begin{array}{l}\text { Leaves (sweet } \\
\text { leaves) }\end{array}$ & Diabetes, Skin disease \\
\hline & Withered Leaves & Leaves \& Fruit & Cold and cough \\
\hline & Withered Leaves & Leaf juice & Menstrual disorder in women \\
\hline & Withered Leaves & $\begin{array}{l}\text { Leaf juice \& Fruit } \\
\text { paste }\end{array}$ & Cold, Cough, Chest diseases \\
\hline Bauhinia racemosa Lam. (Fabaceae) & $\begin{array}{l}\text { Withered Leaves } \\
\text { and Soil }\end{array}$ & $\begin{array}{l}\text { Bauhinia leaf and } \\
\text { Neem (Azadirach- } \\
\text { ta indica A. Juss. } \\
\text { (Meliaceae)) leaf } \\
\text { paste with Thiru- } \\
\text { mani (Sacred soil) }\end{array}$ & $\begin{array}{l}\text { Cough, Cold and other Water } \\
\text { based diseases }\end{array}$ \\
\hline \multirow[t]{2}{*}{ Capparis divaricata Lam. (Capparaceae) } & Withered Bark & Bark paste & Dysentery, Stomach problems \\
\hline & Withered Leaves & Leaf juice with milk & Infertility, Stomach problem \\
\hline \multirow{2}{*}{$\begin{array}{l}\text { Manilkara hexandra (Roxb.) Dubard (Sa- } \\
\text { potaceae) }\end{array}$} & Withered Leaves & Leaf paste & Infertility \\
\hline & Bark & Bark powder paste & Veterinary medicine \\
\hline $\begin{array}{l}\text { Neolamarckia cadamba (Roxb.) Bosser } \\
\text { (Rubiaceae) }\end{array}$ & Withered Bark & $\begin{array}{l}\text { Bark powder de- } \\
\text { coction }\end{array}$ & Rheumatism \\
\hline $\begin{array}{l}\text { Tabernaemontana heyneana Wall. } \\
\text { (Apocynaceae) }\end{array}$ & $\begin{array}{l}\text { Withered Flow- } \\
\text { ers }\end{array}$ & Flowers & Eye diseases \\
\hline \multirow[t]{2}{*}{$\begin{array}{l}\text { Terminalia arjuna (Roxb. ex DC) Wight \& } \\
\text { Arn. (Combretaceae) }\end{array}$} & \multirow[t]{2}{*}{ Withered Bark } & $\begin{array}{l}\text { Bark powder de- } \\
\text { coction }\end{array}$ & Blood pressure \\
\hline & & Bark powder paste & Cut wounds \\
\hline $\begin{array}{l}\text { Terminalia bellirica (Gaertn.) Roxb. } \\
\text { (Combretaceae) }\end{array}$ & Withered Leaves & Leaves (Offerings) & Infertility, Diabetes \\
\hline Vitex negundo L. (Lamiaceae) & Withered Leaves & Leaf decoction & Skin diseases, Cough \\
\hline $\begin{array}{l}\text { Zizyphus mauritiana Lam. (Rhamnace- } \\
\text { ae) }\end{array}$ & Withered Leaves & Leaf paste & Stomachache \\
\hline
\end{tabular}

Most of the devotees use Sthalavriksha plants as a source of medicine in their day to day lives and this knowledge is traditionally taught by elders from their home itself.

\section{Discussion}

Sthalavriksha studies ethnomedicinal uses are scanty. Information on uses of Sthalavrikshas in temples of Tamil Nadu are based on secondary sources (Aravanan 1984, Samy 1978).Thirugnanam (1995) mentioned 69 Sthalavriksha species and their medicinal values, mostly in
Siddha medicine (traditional medicinal practices of Tamil Nadu). Only three research works have been conducted as field studies. Sundara Sobitharaj (1994) recorded 74 plant species including their medicinal uses from 400 temples. Amirthalingam (1998) recorded 60 Sthalavrikshas from 300 temples of Tamil Nadu including traditional medicinal uses. Narasimhan \& Rathnavathy (2003) identified 14 Sthalavriksha species in northern Tamil Nadu. The present study not only reveals a view of Sthalavriksha worship but also the medicinal utilization of Sthalavrikshas. During the present study 112 species were reported 


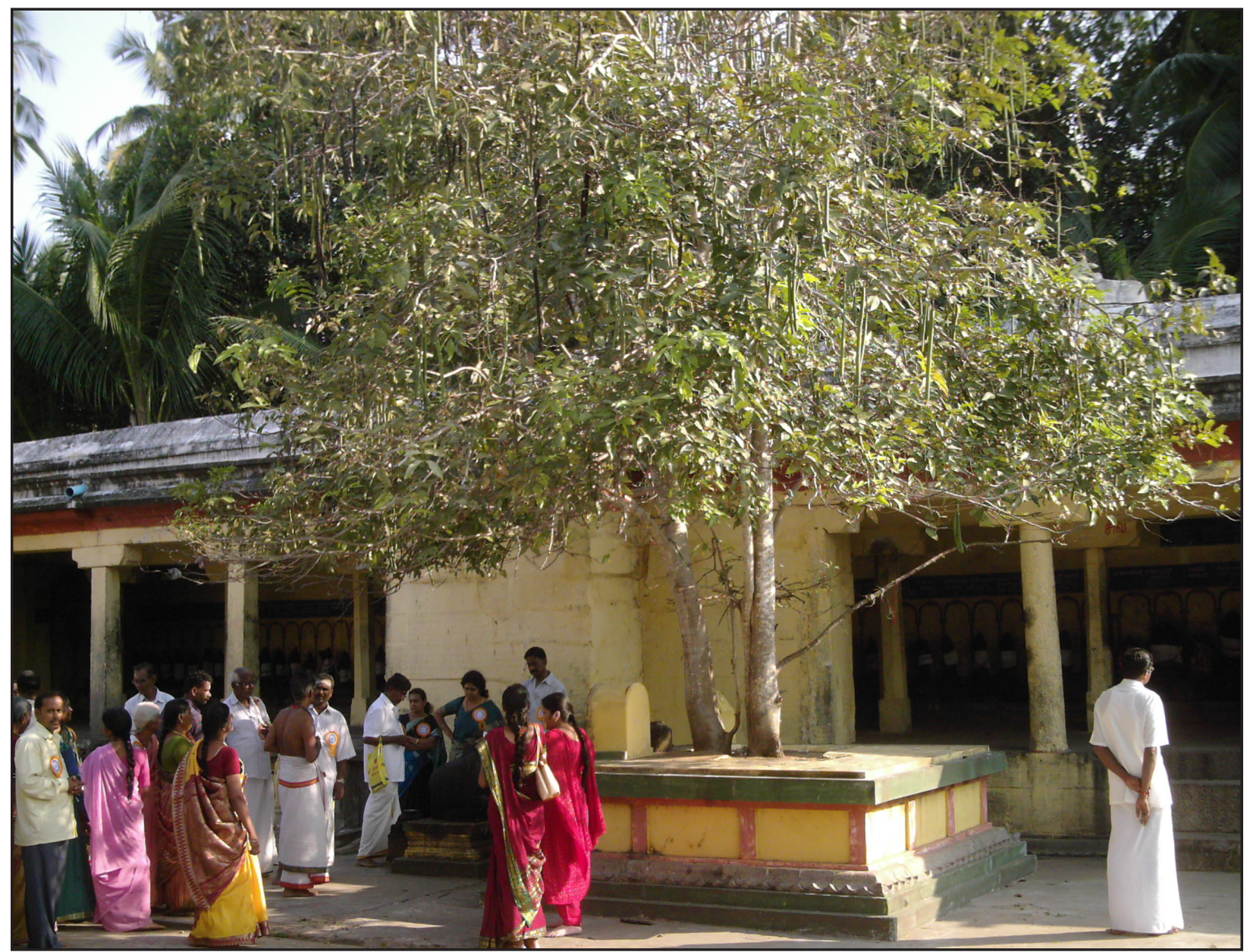

Figure 2. Devotees worshipping Cassia fistula L. at Thiruadigai Siva temple, in Tamil Nadu, India.

as having medicinal uses and 101 medicinal uses Sthalavrikshas were documented. That is to say, $90 \%$ of the Sthalavrikshas are used medicinally.

Worshipping Sthalavrikshas in temples of Tamil Nadu is generally derived from myths and beliefs of the devotees. Sthalapuranas (Temple myth) quotes the stories of demon (Asuras), demi-gods (Devas), saints (Rishis) and several kings who got rid off all the deeds done during previous births (Karma), sin, diseases and ill effects (Dhoshams) by worshipping a deity and performing rituals to the associated Sthalavriksha. Normally, devotees worship the Sthalavrikshas in the temples to obtain boons. Examples maybe: timely marriage, early child birth, extended life span, work for the unemployed, acquiring wealth, reunion of family, relief from diseases, and other such desires. Though the Sthalavrikshas are maintained in the temples of Tamil Nadu for their religious value, they are also utilized for their medicinal purposes. Normally devotees are not allowed to pluck the parts of a Sthalavriksha in a temple, since it is under worship. But on specific occasions, and for medicinal treatment, it is allowed. The devotees and Nattuvaidiyas also collect the withered parts of the Sthalavrikshas in temples. Even if the Sthalavrikshas' parts are consumed for their divine power, these plants are important ingredients used in the traditional Indian medical systems such as Siddha and Ayurveda (Pillai 1931). Of the 112 Sthalavrikshas recorded in Tamil Nadu, Bengal Quince (A. marmelos) was recorded at $40 \%$ of the temples. It is therefore the most frequently occurring Sthalavriksha species in the state. The devotees believe that $A$. marmelos is the most suitable sacred plant to maintain at Lord Siva temple in Tamil Nadu.

Most of the Sthalavrikshas are found in the temple sites even before construction of the temple hence Sthalavriksha worship practice has deeply penetrated into the life style of Tamils and Tamil culture as naming of temple towns, deities and human beings are followed after Sthalavriksha names. An example is Terminalia arjuna (Roxb. ex DC.) Wight \& Arn. It is known as Maruthu in Tamil, based on the Sthalavriksha temple towns known as Maruthamalai, Thirividaimaruthur and Thirupudaimaruthur. Prime deities are named as Maruthasalamurthy and Marutheeswarar. Human beings are named as Maruthamuthu, Maruthi and Maruthai. 


\section{Gunasekaran \& Balasubramanian - Ethnomedicinal uses of Sthalavrikshas (temple trees) in Tamil Nadu, southern India}

The Sthalavrikshas are utilized by devotees for their religious and medicinal values, and are also important germplasm reserve. For instance two threatened species Saraca asoca (Roxb.) De Wilde and Santalum album L. are worshipped as Sthalavriksha. These species are becoming rare in the wild and are included in the Red listed plants of southern India.

Sthalavriksha parts are not only used for their cost effectiveness but also for devotional belief. In certain temples priests also act as traditional healers; prescribing medicines and providing Sthalavriksha leaves as the deity's offering for instance, Acacia farnesiana (L.) Willd. leaves in Melakodumalur temple.

\section{Acknowledgements}

The authors gratefully acknowledge the knowledge providers and user groups including temple priests, devotees and local Nattuvaidyas (traditional medical practitioners) for their help during the field survey. We thank the Commissioner, Department of Hindu Religious and Charitable Endowment, Government of Tamil Nadu for providing permission for temple surveys. We thank the Joint Director, Botanical Survey of India, Southern Circle and the Director, Institute of Forest Genetics \& Tree Breeding, Coimbatore for permitting us to use the herbarium facilities. We thank the director of Salim Ali Centre for Ornithology and Natural History, Anaikatty, Coimbatore, for his encouragement. The authors are thankful to the unknown reviewers for their comments to improve this paper.

\section{Literature Cited}

Amirthalingam, M. 1998. Sacred Trees of Tamil Nadu; A survey. C.P.R. Environmental Education Centre, Chennai, India.

Anonymous, 1988-89. The Wealth of India: Directory of raw materials and industrial products, raw materials, Vol ume 1-11, Publication Directorate, New Delhi.

Anonymous. 1962. Nalayirathiviyaprapantham. Madavadasan, Ramakrishana Mudaliyar, Madras, India.

Aravanan, K.P. 1984. Maravazhipadu Dravida Africa Opeedu. Parinilayam, Chennai, India.

Gamble, J.S. 1915-1936. Reprint, 1986. Flora of the Presidency of Madras, Volume 1-3, Bishen Singh Mahendra Pal Singh, Dehra Dun, India.
Gunasekaran, M. \& P. Balasubramanian. 2005. Sthalavriksha worship; A tool in plant conservation in Tamil Nadu. Pp. 163-166 in Proceedings of National Strategy for Conservation of Sacred Groves. Edited by C. Kunhikannan \& G. Singh. Institute of Forest Genetics and Tree Breeding, Coimbatore, India.

Henry, A.N., G.R. Kumari, \& V. Chitra. 1987. Flora of Tamil Nadu, India, Volume 2. Botanical Survey of India, Coimbatore, India.

Henry, A.N., V. Chitra, \& N.P. Balakrishnan. 1989. Flora of Tamil Nadu, India, Volume 3. Botanical Survey of India, Coimbatore, India.

Nair, N.C. \& A.N. Henry. 1983. Flora of Tamil Nadu, India, Volume 1. Botanical Survey of India, Coimbatore, India.

Narasimhan, D. \& K. Rathnavathy. 2003. Ecology of sacred trees: Temple trees of northern Tamil Nadu. Pondicherry Institute of Linguistics \& Culture Journal of Dravidic Studies 13: 89-118.

Nedunchezhiyan. 2005. Thamizhar Kanda Thavaraviyal. International Institute of Tamil Studies, Chennai, India.

Pillai, S.T.V. 1931. Tamil-English Dictionary of Medicine, Chemistry, Botany and Allied Sciences, Vol I-V, The Research Institute of Siddha's Science, Madras, India.

Samy, B.G.L. 1978. Sources for a history of plant science in India, part IV. Temple- Plants A Reconnaissance. Pp. 23-62 in Transactions of the Archaeological Society of South India, 1965-68. The Archaeological Society of South India, Government Museum, Madras, India.

Srinivasan, K.R. 1972. Temples of South India. National Book Trust, New Delhi.

Sundara Sobitharaj, K.K.S. 1994. Thalamarangal, Sobitham, Chennai, India.

Thambiran, M. 1963. Thayumanavar's Paraparkkani. Kasimadam, Thirupananthal, India.

Thambiran, M. 1997. Thirugnanasambathar Devaram, Volume1-6. Dharumapuram Adeenam, Myladuthurai, India.

Thirugnanam, S. 1995. Thirukoil Marangalin Maruthuvapayangal. Selvi Pathipagam, Tiruchi, India. 
